\title{
Textures Recognition Through Tactile Exploration for Robotic Applications
}

\author{
Samuel Rispal, Axay K. Rana, and Vincent Duchaine \\ Dept. of Automated Manufacturing Engineering \\ École de Technologie Supérieure (ÉTS) \\ Montréal, Québec, Canada \\ e-mail: vincent.duchaine@etsmtl.ca
}

\begin{abstract}
Achieving texture recognition through processing of tactile information could significantly improve robotic prehensile and manipulative capabilities. By producing an object signature based on such information, mishandling due to friction or slippage could be avoided. However, this would require acquisition and processing of tactile data in close to real time in order to function at task speed. This paper proposes a new texture-discriminating algorithm that requires very little exploratory movement. We compared the success rate of two types of exploratory movement for the recognition textures with directional properties such as grooves. Another goal of this study was to obtain an algorithm that is largely insensitive to the velocity and contact force of the sensor movement. We used a genetic algorithm to optimize the variables and the topology of our neural network. We improved the results with a new approach to majority voting that does not require numerous samples. Object classification was more than $90 \%$ correct and most of the errors involved textures that humans are barely able to differentiate.
\end{abstract}

Keywords- Grasping; Tactile intelligence; Tactile sensing; Texture recognition

\section{INTRODUCTION}

The development of tactile sensors has been a very hot topic in the robotic community for decades, since it is obvious that robots with tactile sensory capability would be able to perform more complex tasks [1]. Many recent studies in this subject area have produced impressive results [2], [3], [4]. The tactile sensor is an important link in the tactile perception scheme in a similar way to CCD or CMOS sensors in artificial vision [1]. However, in order to convert this sensory capability into better robotic grasping and manipulation skills, raw signals must be transformed into reliable higher-level information. Tactile sensors are capable of returning considerable amounts of information. Most are sensitive to pressure and can thus provide tactile imagery of normal stresses applied [4], [2], which can represent easily hundreds of data points for a whole robot hand. In order to replicate the human tactile apparatus, several researchers have developed sensors that can also measure shear stress [5], temperature gradient [6] or dynamic information [7]. This high volume and diversity of information makes it difficult to use these sensors in a control algorithm without significant processing [8].

Using tactile sensor signals to recognize object surface textures could significantly improve prehensile and manipu-

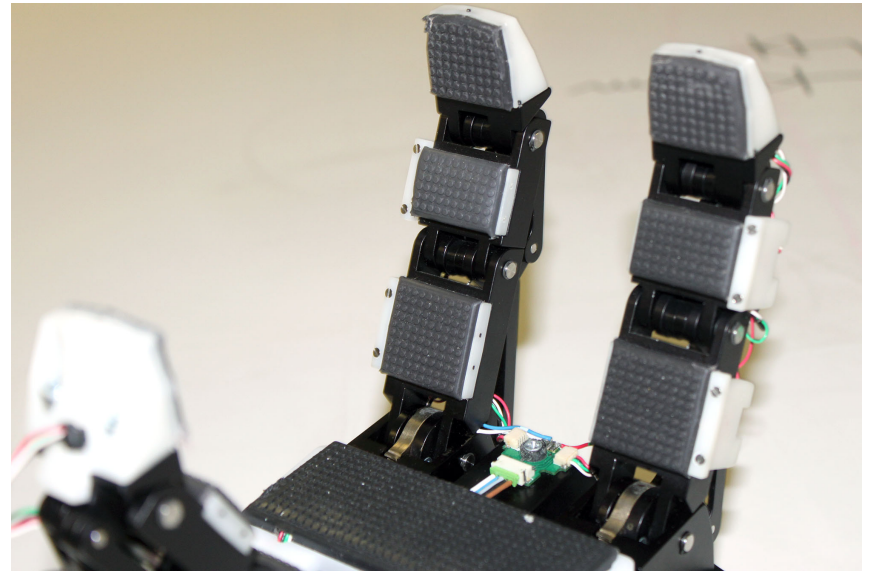

Fig. 1. Tactile sensor used for recognizing texture.

lation skills. Indeed, such information could be used to recognize objects and surface properties thereof such as coefficient of friction in order to develop better grasp strategies. Texture information could also be used to detect possible slippage of a grasped object.

In recent years, some researchers have worked on the use of tactile information for the purpose of recognizing texture. [9] and [10], [11] have thus demonstrated the recognition of considerable numbers of different textures with impressive success rates. The present article describes a signalprocessing learning algorithm that uses the raw dynamic signals of a tactile sensor to recognize object textures. The originality of this work is that the proposed algorithm can achieve after training over $90 \%$ recognition using a single set of data that represent less than one second of acquisition, thus opening the door to real time application. After presenting in section 1 the tactile sensor used in this work, the second section examine different tactile exploration movements for the purpose of making the algorithm insensitive to the directionality of some textures (i.e. parallel grooves). This part of the paper describes also the different features used as inputs to the neural network and the different learning algorithm used. Section 3 then presents an optimization performed using a genetic algorithm to improve texture recognition. The paper conclude by presenting results obtained on 10 different textures from real objects. 


\section{TACTILE SENSOR}

The sensor shown in fig. 1 was used in our experiments. This sensor presented in [12] is a capacitive device able to acquire two types of data, normal stress and dynamic signals.

The first type consist of images of the normal stress applied on the sensor with a resolution of $3 \times 4$ taxels per phalanges. The sampling frequency was set at about $25 \mathrm{~Hz}$ [13]. This capacitive sensor used a two layer microstructured silicone dielectric filled with nanoparticles of ferroelectric ceramic. This characteristic of the sensor allows a very broad measurement range while making it also highly sensitive to low forces $\left(10^{-4} \mathrm{~N}\right.$ per taxel).

The sensor also measures the stress rate applied on the sensor. Instead of differentiating the capacitance value over time, dynamic sensing is achieved here using a transimpedance amplifier that goes out of its equilibrium only in reaction of variation of the sensor capacitance. This make it very sensitive to any dynamic event such as vibration and allow us to have a sampling frequency considerably higher than for static measurements $(1000 \mathrm{~Hz}$ vs $25 \mathrm{~Hz})$. Characterizing the sensor response to dynamic signals is a task more complex than for static load. We used a vibrotactile transducer called the Haptuator [14] and commercialized by TactileLabs to validate the sensitivity of the sensor to vibration both in term of frequency and amplitude. During numerous tests, exceptional sensitivity was observed, exceeding human sensitivity to mechanical vibratory frequency and amplitude. Humans can distinguish frequencies in the $50-500 \mathrm{~Hz}$ range [15], which is the range of acquisition of our sensor. For amplitude, we carried out a simple test in which the Haptuator amplitude was set below the vibrational amplitude detectable by humans (performed on 10 subjects) at $250 \mathrm{~Hz}$. A peak of a clear amplitude on the FFT of the sensor signal was still clearly display.

\section{TEXTURE RECOGNITION ALGORITHMS}

As we have seen, the sense of touch plays a very important role in the manipulation of objects, much more than vision, which allows planning of movement [16]. It is particularly interesting that the manner in which a human seizes or catches an object depends on perception of characteristics such as fragility [17]. Knowing how to evaluate different characteristics of objects touched (texture, fragility, roughness, adhesiveness, etc.) is therefore very relevant to touch robotics. We have chosen to focus on texture discrimination, which could become possible using a sufficiently large database of texture characteristics.

Several studies in this subject area have been published. The first approach to discriminating textures was based on vision [18]. This method underwent many improvements in order to use larger image libraries (use of multi-band images, Gabor filter, etc.) [19] [20]. However, the algorithms became cumbersome and the large number of parameters on which the imaging process depends continues to limit the quality of the results obtained.

In studies based on a tactile approach, sensors based on microphones have been used. Texture discrimination over 90
$\%$ correct has thus been achieved [21]. Two studies in particular attracted our attention: In the first of these, a finger-like probe containing multiple randomly placed sensors (static and dynamic) was used. After data pre-treatment, textures were distinguished using the main components of the Fourier transform in a learning algorithm. Eight textures were differentiated at three different speeds with $90 \%$ success using a majority vote [22] [23].In the second study, a statistical method called Bayesian inference was used, which functions using a relatively small set of training data. However, to confirm a texture, three movements were required. A BioTac probe with static and dynamic sensors (up to $2200 \mathrm{~Hz}$ ) was used. Over $95 \%$ of the 117 textures in the test were recognized [24] but the information acquisition step required a relatively long time.

Various sensor technologies such as MEMS [25] [26] and opto-tactile sensors [27] have been tested in other studies.

The goal of our study is to identify suitable texture acquisition movements and a lightweight algorithm that would open the door to real-time applications during object manipulation or exploration of an environment. For this, we have focused on three elements: $i$ the choice of movement, ii. data acquisition, pre-processing and extraction, and iii. learning algorithms. The figure 2 represents the steps of the algorithm.

\section{A. Exploratory motions}

The exploratory movement has a major impact on the quality of texture discrimination. Several studies have examined the implications of the choice of movement. Twodimensional linear motion has been chosen in many cases [23] [28]. Object typing is another approach that been studied [29]. Finally, a thorough study of human texture recognition movements has been published [24].The authors selected three movements in an attempt to discriminate textures using fewer learning data and very-high-success-rate data.

For the purposes of our study, we selected two exploratory movements. The first is a short sliding linear movement in a single direction. This movement can be compared to the friction of the thumb on an object in the hand. One of the challenges of this movement is to distinguish textures at variable speed and pressure. If this can be achieved, it will become possible to characterize slippage, for example to differentiate the slippage of an object in the hand from rubbing of the object against another object. The second exploratory movement is a circular motion against a flat surface at constant speed and force. This movement is similar to the movement made by a human with the index and middle fingers to determine the roughness of a surface. This movement is particularly interesting because it should allow better discrimination between textures that are not similar in all directions (grooves, concentric circles, etc.).

Figure 3 illustrates these two movements.

\section{B. Features}

The choice of discriminants is particularly important since they determine the quality of discrimination, as does the 


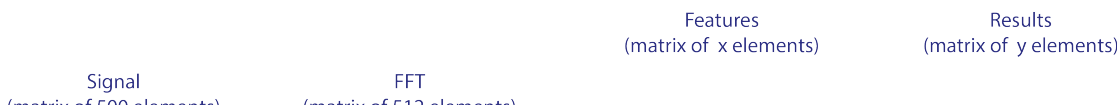

(matrix of 500 elements) (matrix of 512 elements)

(5 integer if majority voting)
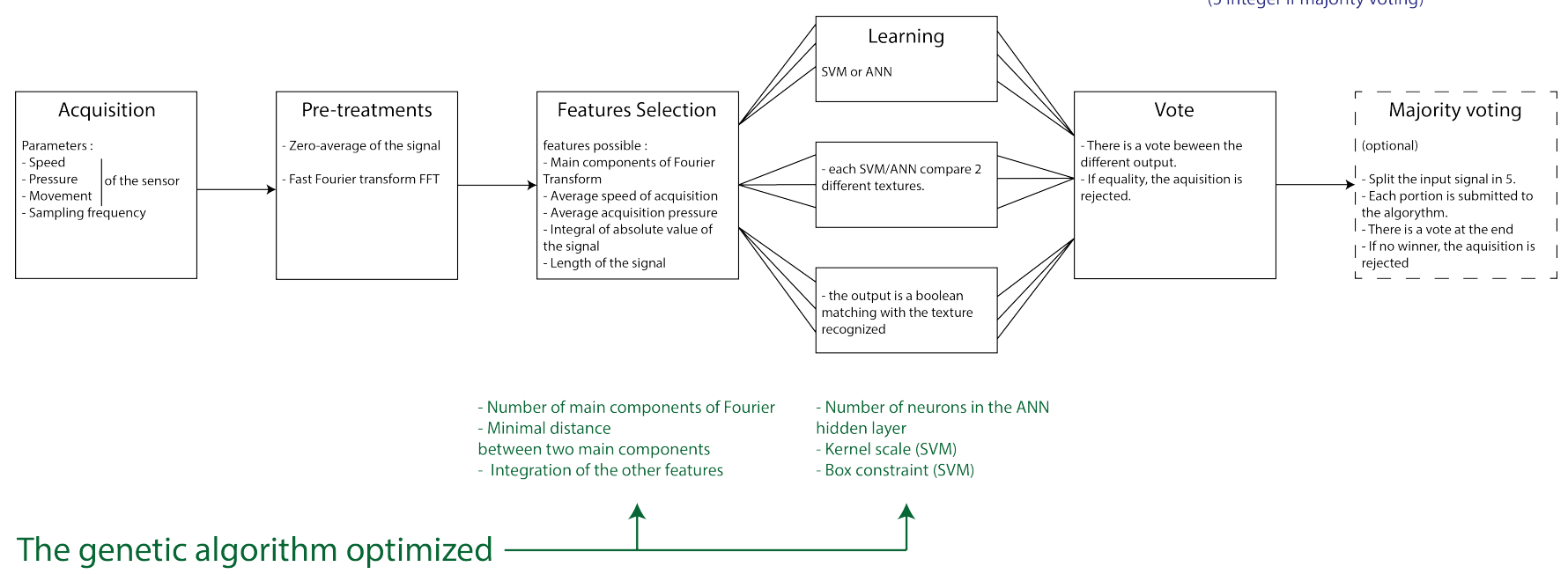

Fig. 2. Steps of the algorithm

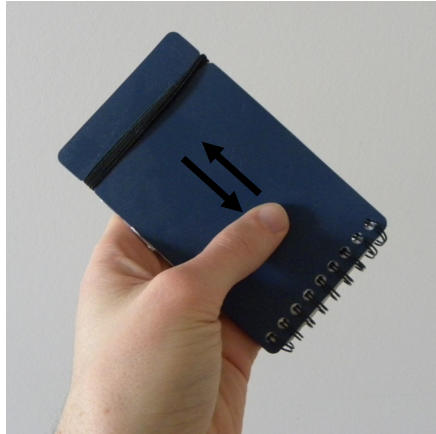

a) short sliding linear movement

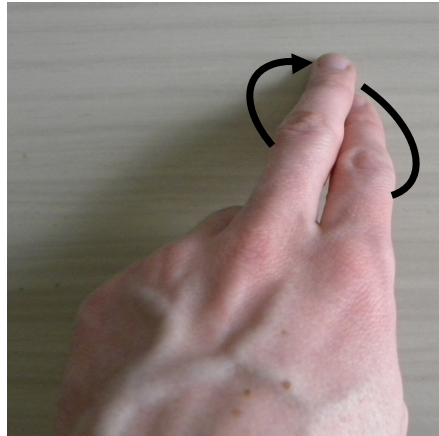

b) circular exploration movement

Fig. 3. Illustration of the two exploratory movements

selection of movements. Before extracting discriminators, two pre-treatments are applied. The signal is first zeroaveraged in order to obtain a Fourier transform with no null frequency component. The fast Fourier transform (FFT) that will extract the discriminants is then calculated in postprocessing Matlab using the function fft. The duration of the input signal is from $450 \mathrm{~ms}$ to $600 \mathrm{~ms}$. The Fourier transform is calculated on 1024 values distributed equally between 0 $\mathrm{Hz}$ and $500 \mathrm{~Hz}$. The figure 4 show the signal and its Fourier transform.

To select discriminants, most studies using capacitive sensors or microphones have drawn inspiration from sound processing and used the main components of the Fourier transform of the maxima using detection algorithms. Several studies have also taken into account the speed and the force applied by the robot. In our case, we will study the impact of several discriminants and let the genetic algorithm determine whether or not to use them or to change their parameters. The discriminants and their features are described below:

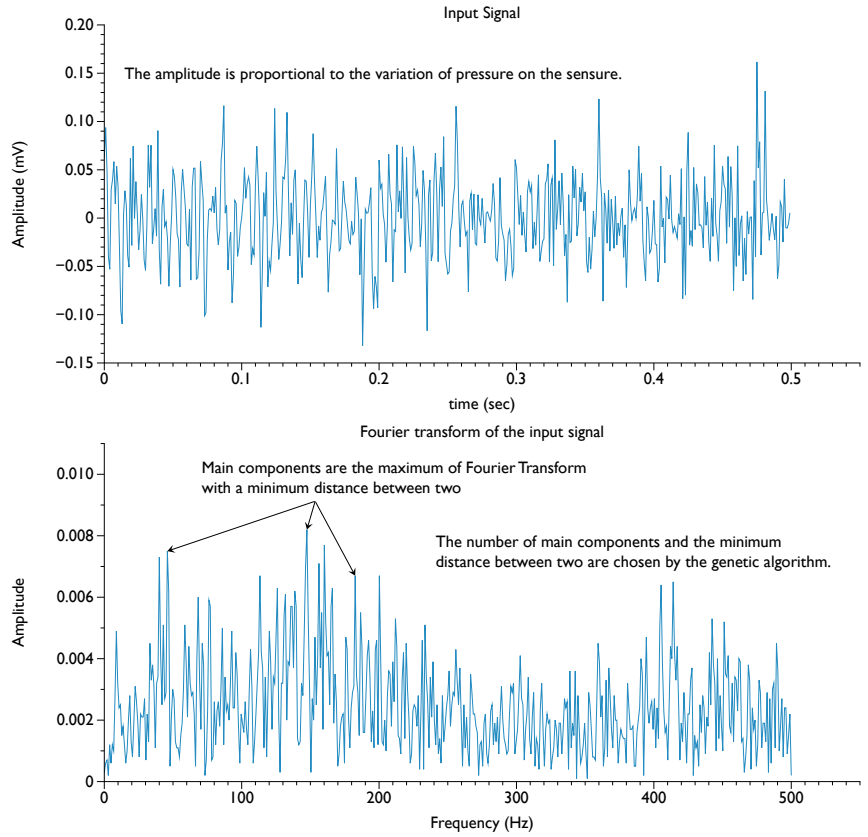

Fig. 4. Graphic of the input signal and its Fourier transform

The main components of the Fourier transform: These components show the predominant frequencies in the material, which are related directly to the material surface properties (lines, grid, etc.) and are somewhat correlated with the speed of acquisition. Our algorithm detects the maximum in the Fourier transform. A tolerance margin imposes a minimal gap between the maxima. The user (or the genetic algorithm) selects the number of detected maxima and the code uses the frequency and amplitude of these peaks as the discriminant of the learning algorithm.

The average speed of acquisition: An estimate of 
the average speed of acquisition (scanning) is included in the learning algorithm in order to improve anticipation of changes in peak Fourier transform frequency.

The average acquisition pressure: The force applied to the sensor influences the amplitude of the various peaks of the Fourier transform.

The integral of the absolute value of the signal $i$ is calculated using equation 1 :

$$
i=\int_{0}^{l}\left|s(t)^{2}\right| \mathrm{d} t
$$

In this formula, $l$ is the signal length and $s(t)$ represents the signal values. This value allows better understanding of the signal and can be brought in line with the average force. It should allow differentiation of two signals that vary in intensity and low frequencies.

Signal length $l$ is calculated by the equation 2 .

$$
l=\sum_{k=1}^{l} \sqrt{\left(t_{k+1}-t_{k}\right)^{2}+\left(s_{k+1}-s_{k}\right)^{2}}
$$

This index also provides information on signal amplitude and frequency.

\section{Neural Network}

The discriminants are then submitted to a learning algorithm. The learning algorithms selected to process the discriminants are among the most popular: artificial neural networks (ANN) and support vector machines (SVM). For ANN, we used the multilayer perceptron with the number of inputs and the number of neurons in the hidden layer depending on the genetic algorithm. The transfer function in the hidden layer is sigmoid and the output neuron is a linear function. Following this, a rounding function provides a Boolean output comparing two different textures. To differentiate more than two textures, we used a set of ANN (or SVM) each representing the differentiation between two paired textures. The texture output from such an ANN (or SVM) assembly is that determined by the largest number of ANN (or SVM). If the result is a tie between two textures, the output is considered unclassified.

Creating an ANN or SVM for each pairing of textures can involve creating large numbers of neuronal networks: combinations of 2 from $n$ choices, with $n=$ the number of textures. This number is $n ! / 2(n-2)$ !, that is, six in order to differentiate four textures, 45 in order to differentiate 10 textures, and so on. Using ANN or SVM to compare textures in pairs provides better understanding of the challenge of differentiating two textures and adapting the learning algorithm as a function of texture (with the genetic algorithm). However, this becomes very onerous if too many textures are involved.

There are then two ways to perform the learning task: the first involves extracting the discriminants from the entire input and running them through the learning algorithm. The second way is to obtain the largest majority vote among the five responses obtained by dividing the acquired data into five samples, extracting the discriminant and performing the learning task independently on each of these samples. This type of algorithm improves overall performance and has been used previously with success [23][24]. But in the previous cases, instead of dividing the data, they used many different data.

\section{OPTIMIZING WITH THE GENETIC ALGORITHM}

Many algorithm variables must be optimized for an optimal result (ANN or SVM configuration, number of main FFT components, choice of discriminators, etc.). For this purpose, we used a standard genetic algorithm (GA) with 20 individuals and 30 generations.

For each individual, the algorithm separates the database into two equal parts: a learning portion, and a testing portion (to limit over-fitting). The algorithm performs 20 different learning tasks, which are then tested with the test database. The fitness function returns the average of the results of learning and testing.

The GA determines a set of variables for each exploratory movement algorithm, that is, the short linear slide movement (SLSM) and the circular exploration movement (CEM). The variables are listed below:

1) Number of frequency peaks (SLSM/CEM): The value of this variable ranges between 2 and 15, and indicates the number of relevant peaks required to define the texture.

2) Minimum frequency between each peak (SLSM/CEM): This value varies between 5 and $50 \mathrm{~Hz}$. The purpose of the spread is to filter noise produced by adjacent peaks but without having an impact on major peaks.

3) Ranking by frequency or intensity (SLSM/CEM): This variable determines if the classification of peaks must be by intensity or frequency before entry into the learning algorithm.

4) Integration of average sensor speed on the object surface (SLSM):This variable has three options: nonintegration of the average speed of the sensor, integration as a variable of the learning algorithm, or integration as time stretching allowing all acquisitions at the same speed $(1 \mathrm{~s}$ at $1 \mathrm{~m} / \mathrm{s}$ becomes $0.5 \mathrm{~s} 2 \mathrm{~m} / \mathrm{s})$.

5) Integration of the average force applied by the sensor on the object surface (SLSM): This variable is used to determine whether or not force should be included as variable input to the learning algorithm.

6) Signal integral (SLSM /CEM): Used to determine whether or not the signal integral should be used as an input variable of the learning algorithm.

7) Signal length (SLSM /CEM): Used to determine whether or not signal length should be included as variable input of the learning algorithm.

8) Rotary element decomposition (CEM): Since the exploration movement is rotary, the acquired data may be divided into one to nine parts and Fourier peaks extracted from each sub-acquisition.

The last three variables are learning algorithm variables: 
1) Number of neurons in the ANN hidden layer (SLSM/CEM): This varies between 5 and 50 .

2) Box constraint (SVM): This ranges between $10^{-} 2$ and 10. This value limits the size of certain SVM vector coefficients and thereby establishes different borders.

3) Kernel scale (SVM): This ranges between $10^{-} 5$ and $10^{5}$. This variable changes the Kernel function scale, thus allowing non-linear transformations.

To change the last two parameters, we followed the recommendations provided in the Help document in Matlab.

\section{A. Methodology}

To investigate the influence of different movements on texture perception, we propose two experiments, one to study the impact of sensor speed and pressure associated with linear movement, and another to determine the influence of the initial orientation of the texture by comparing linear and rotary movements.

In the first experiment (SLSM), we compared the four textures shown in Figure 5. Although the amount of texture involved in this experiment is low compared to some other studies, these ones are all very similar and therefore very hard to discriminate. We could have use a number of textures lot higher without never running into such challenge. Our assumption is that if we can solve the problem with these very close textures we should be able to easily discriminate texture that are much more different. These textures present groove or grid patterns very similar to one another. For example, the difference between textures Ridge $2 \mathrm{a}$ and Ridge $2 b$ is only the depth of the grooves.

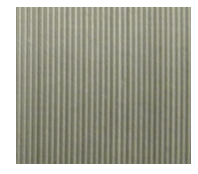

Ridge 1

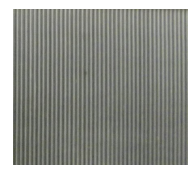

Ridge 2a

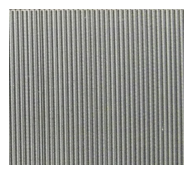

Ridge $2 b$

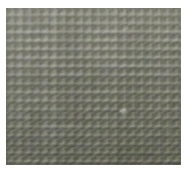

Grid 1
Fig. 5. Texture for the SLSM experiment

We then acquired the data at three different speeds and three different pressures. Fifty scans were obtained per texture, speed and pressure. This represents 450 samples per texture, representing a database of 1800 samples. The duration of each scan was 500 milliseconds. This time made it possible to scan $10 \mathrm{~cm}$ of texture at the fastest speed. Average speeds selected were $0.1 \mathrm{~m} / \mathrm{s}, 0.15 \mathrm{~m} / \mathrm{s}$ and $0.2 \mathrm{~m} / \mathrm{s}$ and the forces applied were $1 \mathrm{~N}, 2.5 \mathrm{~N}$ and $5 \mathrm{~N}$.

For the second experiment, we sought to distinguish the five textures shown in Figure 6. They feature very slight variations in groove and grid characteristics.

We sought to determine a scanning movement and algorithm capable of recognizing a texture whether in line or diagonally with the grooves. To achieve this, we compared the two exploratory movement types shown in Figure 3. The first type is a unidirectional linear gesture at constant speed and average force over a distance of about $5 \mathrm{~cm}$. The

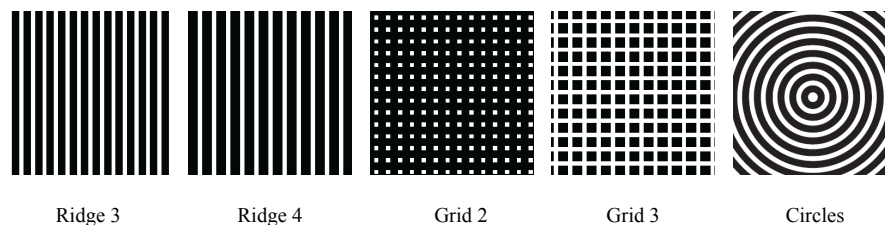

Fig. 6. Texture for the CEM experiment

movement is executed at different orientations to the texture: 0 degrees, 30 degrees, 45 degrees and 90 degrees relative to the grooves. The second type is a circular movement with a radius of about 2. Data are acquired at 0 degrees, 30 degrees, 45 degrees and 90 degrees relative to the grooves.

For each exploratory movement, 200 scans were obtained per texture. For the unidirectional movement, scans were separated in 4, 50 scans with different orientations ( 0 degrees (with the grooves), 45, 90 and 30 degrees). For circular motion, the starting point was changed. The same four angles were used as a starting point.

\section{B. Results}

The dataset is separated in two categories : the learning samples and the test samples. The first set feeds the learning algorithm. The second set tests the learning independently of the first one.

1) Linear movement at variable speed and force: The simulation required eight hours. Recognition of the four textures was $82.0 \%$ correct with $8.5 \%$ rejection (learning: $85.1 \%$ with $5.9 \%$ rejection; test: $78.7 \%$ with $8.8 \%$ rejection) for ANN and $85.3 \%$ with $7.8 \%$ rejection (learning: $94.8 \%$ with $4.5 \%$ rejection; test: $75.0 \%$ with $11 \%$ rejection) for SVM. Textures in pairs were differentiated correctly $85.7 \%$ to $93.8 \%$ of the time by ANN (learning set: 87.8-96.2 \%, testing set: 81.8-93.5\%). For SVM, this was $86.4 \%$ to $94.8 \%$ (learning set: $87.1-100 \%$, testing set: 77.6-94\%). The errors were relatively well distributed, even though texture 4 was less well recognized, which was not unexpected since it has a grid rather than groove pattern and was therefore quite different. The challenge of optimizing the ANN was to differentiate textures 2 and 3 , which are very similar. Human perception have difficulties to differentiate both.

It should be noted that the optimized variables resulting from the genetic algorithm were separated into two groups. Some varied considerably while others did not. For neural networks, the number of Fourier transformation peaks required to differentiate two textures varied between 3 and 15 textures depending on the two materials being differentiated. Similarly, the number of neurons in the hidden layer varied considerably in general. Furthermore, it is interesting to note that scanning speed, the integral and the length of the signal were used extensively while pressure was ultimately used less (50\% of cases). Finally, speed was used most often to relax the time rather than directly as input to the ANN. In the case of the SVM, the number of peaks was less variable. 
The average speed was still used, but in contrast, pressure, the integral and signal duration were less used.

Using the majority vote mode, the $500 \mathrm{~ms}$ scans were separated into five $100 \mathrm{~ms}$ portions. Each mini-acquisition was submitted to the optimized ANN or SVM. The answer that received at least three votes determined the response assigned to the scan. In this case, optimization took much longer since the database was much larger. To reduce the acquisition time, we performed a small-scale optimization with 10 individuals over 10 generations starting from the previous simulation result. In this mode, the ANN reached $84.4 \%$ recognition with $5.6 \%$ rejection (learning: $84.9 \%$ with $5.6 \%$ rejection; test: $84.0 \%$ with $5.4 \%$ rejection)(an improvement of $2.2 \%$ ), while the SVM reached $98.2 \%$ recognition with $0.9 \%$ rejection (learning: $99.1 \%$ with $0.2 \%$ rejection; test: $97.3 \%$ with $1.6 \%$ rejection)(an improvement of $12.9 \%$ ). Table I 1 shows the SVM confusion matrix. We note that the two elements that caused the most errors were the second and third. Nevertheless, the quality of acquisition may also have an impact, as we will see later.

TABLE I

CONFUSION MATRIX OF SLSM WITH SVM LEARNING AND MAJORITY VOTING (5 VOTES)

\begin{tabular}{|c|c|c|c|c|c|}
\hline \multirow{5}{*}{$\begin{array}{ll}\text { Ridge 1 (450) } \\
\text { Ridge 2a (450) } \\
\text { Ridge 2b (450) } \\
\text { Grid 1 (450) }\end{array}$} & $\begin{array}{l}\overline{0} \\
\overrightarrow{0} \\
\overrightarrow{0}\end{array}$ & 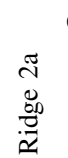 & 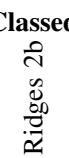 & $\overline{7}$ & 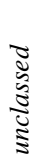 \\
\hline & 445 & & 2 & 2 & 1 \\
\hline & 1 & 427 & 15 & 1 & 6 \\
\hline & & 4 & 437 & 2 & 7 \\
\hline & 1 & 2 & 2 & 443 & 2 \\
\hline
\end{tabular}

2) Linear movement vs rotating movement: Learning was performed using a genetic algorithm with 20 individuals over 30 generations. We note with interest that for the first type of movement, the ANN provided $76.3 \%$ recognition with $13.1 \%$ rejection (learning: $80.9 \%$ with $11 \%$ rejection; test: $71.5 \%$ with $14.2 \%$ rejection) and the SVM provided $87.0 \%$ with $7.8 \%$ rejection (learning: $96.3 \%$ with $2.2 \%$ rejection; test: $76.4 \%$ with $13.4 \%$ rejection). The textures that were difficult to differentiate were $(a, b)$ and $(b, d)$. In the case of the latter, the patterns were different (grooves and grid), while the frequency components were the same. These results are nevertheless very good. The greatest loss of information occurred because scanning in the direction of the grooves contained very few frequency components. In the case of circular movement, the algorithm divided the acquisition into portions (numbering 1, 3, 5, 7 or 9) and submitted them individually to the ANN or SVM. A vote was then obtained to determine which texture was most likely. This provided the following results. Recognition by the ANN was $82.9 \%$ with $2.0 \%$ rejection rejection (learning: $84.6 \%$ with $1.2 \%$ rejection; test: $81.1 \%$ with $2.8 \%$ rejection) and by the SVM, $93.0 \%$ with $3.4 \%$ rejection (learning: $99.0 \%$ with $1.6 \%$ rejection; test: $86.7 \%$ with $5.2 \%$ rejection). We thus observed a marked increase but also an interesting reduction in rejection. However, it remained difficult to differentiate textures (a) and (b). Figure II shows the confusion matrix for an SVM.

TABLE II

CONFUSION MATRIX OF CEM WITH SVM AND A ROTATING EXPLORATORY MOVEMENT

\begin{tabular}{|c|c|c|c|c|c|c|}
\hline & & & Classe & & & \\
\hline & $\begin{array}{l}\overrightarrow{0} \\
\overrightarrow{0} \\
\vec{\alpha}\end{array}$ & $\begin{array}{l}N \\
\mathbb{D} \\
\mathbb{\infty} \\
\mathbb{\alpha}\end{array}$ & $\frac{N}{3}$ & $\frac{m}{3}$ & $\frac{\mathscr{U}}{\mathscr{U}}$ & 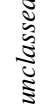 \\
\hline Ridge 1 & 157 & 30 & & & 2 & 11 \\
\hline Ridge 2 & 28 & 162 & & 1 & 6 & \\
\hline Grid 2 & 2 & & 192 & 1 & & 5 \\
\hline Grid 3 & 1 & & & 190 & 1 & 8 \\
\hline Circles & 1 & & & 1 & 197 & 1 \\
\hline
\end{tabular}

We note that the trend was to divide the acquired data into 7 or 9 portions in almost all cases. Six or seven ANN out of 10 used the integral of the signal and its duration. The number of main FFT components and neurons in the hidden layer was very variable. The SVM tended to use primarily the integral and the signal duration. The SVM coefficients (BoxConstraint and KernelScale) were generally constant and relatively high.

In conclusion, circular movement allowed a considerable increase in recognition compared to simple linear movement. This movement could be used to explore relatively large environments and flat surfaces (greater than $5 \times 5 \mathrm{~cm}$ ). However, linear movement should not be overlooked, given that it also has many applications: it allows touching a hand-held object and can be more discrete during a manipulation. The movement can be chosen according to the time available to the robot and the type of desired result: circular movement provides more reliable results but requires dedicated exploration time, while linear movement, although its results are more uncertain, is faster and has more applications.

\section{EXPERIMENT ON REAL LIFE TEXTURES}

In both of the above experiments, we worked with textures that were crafted by hand and relatively rough. Before conducting additional experiments based on the intrinsic properties of the materials, it was important to verify that the sensors were functional with fine textures. For this experiment, we tested our algorithm with 10 different textures of which four were very fine. We chose the optimal conditions: controlled velocity and force, textures having little variation with respect to rotation.

\section{A. Experimental Protocol}

The goal of this experiment was to use the high resolution of our sensor to differentiate fine textures that resemble each other. Ten common textures (fig. 7) encountered in daily life were used.

For each texture, 200 pieces of data were collected at constant average velocity and force. The sensor rubbed over a distance of $10 \mathrm{~cm}$ at $125 \mathrm{~mm} / \mathrm{s}$ with a force of about $2 \mathrm{~N}$. 
TABLE III

CONFUSION MATRIX OF THE SVM OF 10 FINE TEXTURES

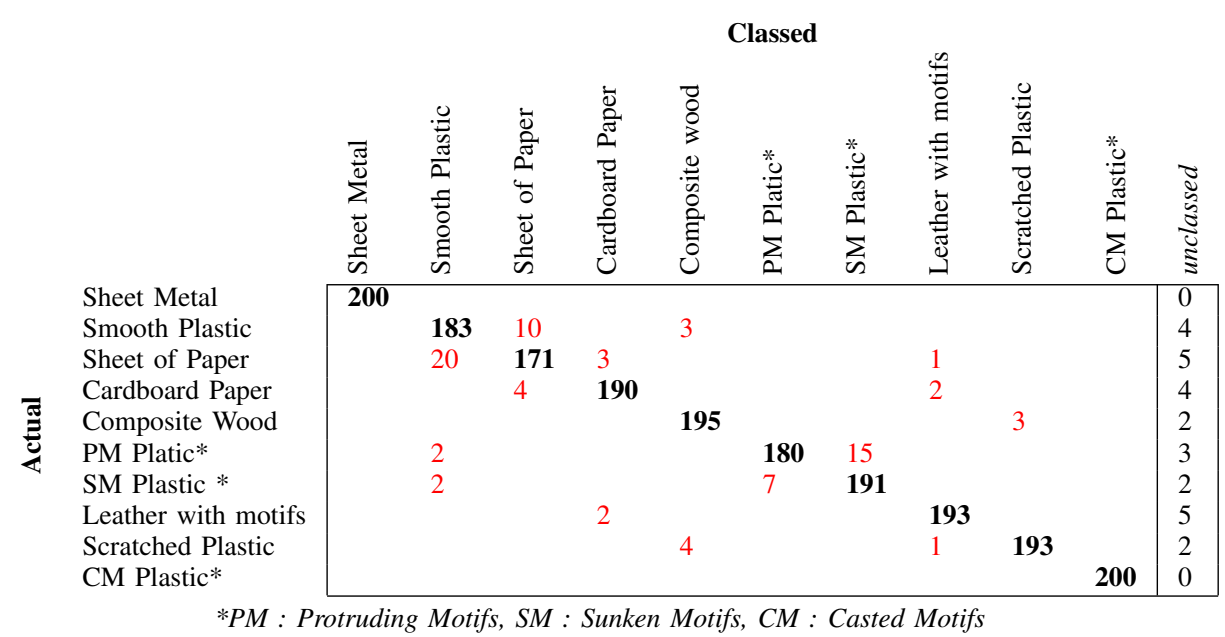

TABLE IV

SUMMARY TABLE OF THE RESULTS

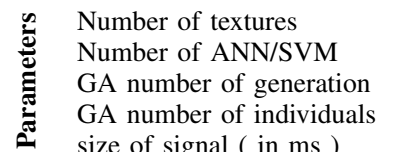

GA number of individuals

size of signal ( in ms ) acquisition frequency number of acquisitions per texture

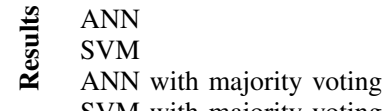
SVM with majority voting

\begin{tabular}{|c|c|c|c|c|c|}
\hline \multicolumn{2}{|c|}{ SLSM } & \multicolumn{2}{|c|}{ CEM } & \multicolumn{2}{|c|}{ Real life textures } \\
\hline \multicolumn{2}{|c|}{4} & \multicolumn{2}{|c|}{5} & \multicolumn{2}{|c|}{10} \\
\hline \multicolumn{2}{|c|}{6} & \multicolumn{2}{|c|}{10} & \multicolumn{2}{|c|}{45} \\
\hline \multicolumn{2}{|c|}{30} & \multicolumn{2}{|c|}{30} & \multicolumn{2}{|c|}{30} \\
\hline \multicolumn{2}{|c|}{20} & \multicolumn{2}{|c|}{20} & \multicolumn{2}{|c|}{20} \\
\hline \multicolumn{2}{|c|}{500} & \multicolumn{2}{|c|}{500} & \multicolumn{2}{|c|}{800} \\
\hline \multicolumn{2}{|c|}{1000} & \multicolumn{2}{|c|}{1000} & \multicolumn{2}{|c|}{1000} \\
\hline \multicolumn{2}{|c|}{450} & \multicolumn{2}{|c|}{200} & \multicolumn{2}{|c|}{200} \\
\hline learning & test & learning & test & learning & test \\
\hline $85.1 \%$ & $78.7 \%$ & $84.6 \%$ & $81.1 \%$ & $96.3 \%$ & $94.9 \%$ \\
\hline $94.8 \%$ & $75.0 \%$ & $99.0 \%$ & $86.7 \%$ & $96.0 \%$ & $95.8 \%$ \\
\hline $84.9 \%$ & $84.0 \%$ & & & & \\
\hline $99.1 \%$ & $97.3 \%$ & & & & \\
\hline
\end{tabular}

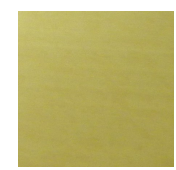

Sheet Metal

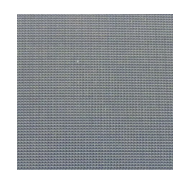
Protruding Motifs
Plastic

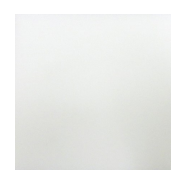

Smooth Plastic

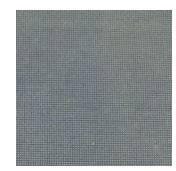

Sunken Motifs Plastic

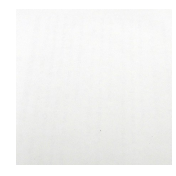

Sheet of Paper

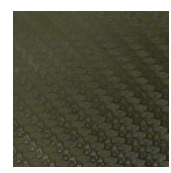

Leather with motifs

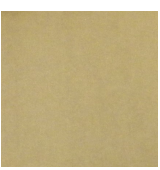

Cardboard Paper

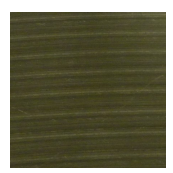

Scratched Plastic

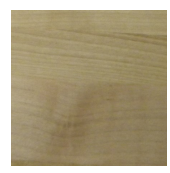

Composite Wood

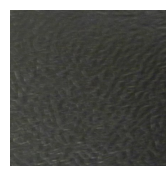

Casted Motifs Plastic
Fig. 7. Textures for the experiment of sensibility

\section{B. Results}

The architecture used contained 45 ANN or SVM and differentiated textures in pairs. As with the method used above, the texture that was identified by the greatest number of ANN or SVM was selected as the correct identification. In the case of equality, the data were considered unclassified. In order to optimize the large networks, we used a genetic algorithm with 10 generations and 13 individuals. Each optimization was based on the results of the SLSM experiment. It lasted eight hours and did not include the majority vote algorithm. The results are very promising: $95.6 \%$ with $0.6 \%$ rejected (learning: $96.3 \%$ with $0.5 \%$ rejection; test: $94.9 \%$ with $0.7 \%$ rejection) for the ANN and $95.8 \%$ with $1.4 \%$ rejected (learning: $96.0 \%$ with $1.2 \%$ rejection; test: $95.8 \%$ with $51.6 \%$ rejection). Using pairings, recognition was at least $98.5 \%$ and most can differentiate $100 \%$ of the textures. Table III presents the confusion matrix for the SVM.

We can see that the two main sources of error are the smooth plastic and the sheet of paper as well as the plastic with protruding pattern and the plastic with sunken pattern. In the first case, the sensor tended to stick to the smooth plastic. This no doubt produced the false data that made the plastic resemble the paper surface. In the second case, the textures are relatively similar and certain scans were likely of fair quality. The errors nevertheless remained below the $10 \%$ threshold.

\section{CONCLUSION}

The results of this study, summarised on table IV, are satisfactory, with overall $96 \%$ recognition of the textures tested. Similar performance has been achieved in other studies, but our sensor and algorithm display the potential to function with four or five textures or more [21][30].

The innovative aspect appears in the first two experiments. The first one compared samples scanned at different velocity and pressure. Recognition was greater than $98 \%$ thanks 
to the proposed way of conducting majority voting. This consists of dividing the data sample into portions instead of using different samples. Misclassification in this study occurred in the case of very similar textures. We suggest that generalizing to a wide variety of textures could work very well. The second experiment examined the effectiveness of a new movement to reduce variance due to orientation. Recognition reached $93 \%$ for five textures, while misclassification was due to difficulty in discriminating between grooved textures. This suggests that it will be complicated to use this movement to differentiate textures of very similar form. Using a genetic algorithm is new in the texture discrimination field and our study shows that it can be useful and powerful. It supports many different tests and improves results significantly. The only problem is the long simulation time. Our study proposes a new approach to shorter acquisition time and raises the possibility of real-time application. The next question we should answer is how to use it to differentiate different frictions or improve object manipulations.

\section{ACKNOWLEDGEMENT}

The authors would like to acknowledge NSERC of Canada for funding and resources.

\section{REFERENCES}

[1] M. R. Cutkosky, R. D. Howe, and W. R. Provancher, "Force and tactile sensors," Springer Handbook of Robotics, pp. 455-476, 2008.

[2] A. Schmitz, M. Maggiali, L. Natale, B. Bonino, and G. Metta, "A tactile sensor for the fingertips of the humanoid robot icub," in Intelligent Robots and Systems (IROS), 2010 IEEE/RSJ International Conference on. IEEE, 2010, pp. 2212-2217.

[3] J. Ulmen and M. Cutkosky, "A robust, low-cost and low-noise artificial skin for human-friendly robots," in Robotics and Automation (ICRA), 2010 IEEE International Conference on. IEEE, 2010, pp. 4836-4841.

[4] R. Li, R. Platt Jr, W. Yuan, A. t. Pas, N. Roscup, M. A. Srinivasan, and E. H. Adelson, "Localization and manipulation of small parts using gelsight tactile sensing," 2014.

[5] K. Noda, K. Hoshino, K. Matsumoto, and I. Shimoyama, "A shear stress sensor for tactile sensing with the piezoresistive cantilever standing in elastic material," Sensors and Actuators A: physical, vol. 127, no. 2, pp. 295-301, 2006.

[6] Y.-J. Yang, M.-Y. Cheng, W.-Y. Chang, L.-C. Tsao, S.-A. Yang, W.-P. Shih, F.-Y. Chang, S.-H. Chang, and K.-C. Fan, "An integrated flexible temperature and tactile sensing array using pi-copper films," Sensors and Actuators A: Physical, vol. 143, no. 1, pp. 143-153, 2008.

[7] M. R. Cutkosky and J. Ulmen, "Dynamic tactile sensing," in The Human Hand as an Inspiration for Robot Hand Development. Springer, 2014, pp. 389-403.

[8] D. Goger, N. Gorges, and H. Worn, "Tactile sensing for an anthropomorphic robotic hand: Hardware and signal processing," in Robotics and Automation, 2009. ICRA'09. IEEE International Conference on. IEEE, 2009, pp. 895-901.

[9] W. Mayol-Cuevas, J. Juarez-Guerrero, and S. Munoz-Gutierrez, "A first approach to tactile texture recognition," in Systems, Man, and Cybernetics, 1998. 1998 IEEE International Conference on, vol. 5. IEEE, 1998, pp. 4246-4250.

[10] J. A. Fishel and G. E. Loeb, "Bayesian exploration for intelligent identification of textures," Frontiers in neurorobotics, vol. 6, 2012.

[11] D. Xu, G. E. Loeb, and J. A. Fishel, "Tactile identification of objects using bayesian exploration," in Robotics and Automation (ICRA), 2013 IEEE International Conference on. IEEE, 2013, pp. 3056-3061.

[12] A. Rana, J.-P. Roberge, and V. Duchaine, "An improved soft dielectric for a highly sensitive capacitive tactile sensor," IEEE Sensors Journal, vol. 16, no. 22, pp. 7853-7863, 2016.

[13] A. Rana and V. Duchaine, "Improved soft dielectric for highly sensitive capacitive tactile sensor," in Robotics and Automation (ICRA), 2013 IEEE International Conference on (Workshop on tactiles sensors). IEEE, 2013.
[14] W. McMahan and K. J. Kuchenbecker, "Dynamic modeling and control of voice-coil actuators for high-fidelity display of haptic vibrations," in Haptics Symposium (HAPTICS), 2014 IEEE. IEEE, 2014, pp. 115-122.

[15] A. B. Vallbo and R. Johansson, "Properties of cutaneous mechanoreceptors in the human hand related to touch sensation," Hum Neurobiol, vol. 3, no. 1, pp. 3-14, 1984.

[16] R. S. Johansson and J. R. Flanagan, "Coding and use of tactile signals from the fingertips in object manipulation tasks," Nature Reviews Neuroscience, vol. 10, no. 5, pp. 345-359, 2009.

[17] G. Savelsbergh, B. Steenbergen, and J. Van der Kamp, "The role of fragility information in the guidance of the precision grip," Human Movement Science, vol. 15, no. 1, pp. 115-127, 1996.

[18] A. Khotanzad and R. L. Kashyap, "Feature selection for texture recognition based on image synthesis," Systems, Man and Cybernetics, IEEE Transactions on, vol. 17, no. 6, pp. 1087-1095, 1987.

[19] A. Jain and G. Healey, "A multiscale representation including opponent color features for texture recognition," Image Processing, IEEE Transactions on, vol. 7, no. 1, pp. 124-128, 1998.

[20] M. Shi and G. Healey, "Hyperspectral texture recognition using a multiscale opponent representation," Geoscience and Remote Sensing, IEEE Transactions on, vol. 41, no. 5, pp. 1090-1095, 2003.

[21] W. Mayol-Cuevas, J. Juarez-Guerrero, and S. Munoz-Gutierrez, "A first approach to tactile texture recognition," in Systems, Man, and Cybernetics, 1998. 1998 IEEE International Conference on, vol. 5. IEEE, 1998, Conference Proceedings, pp. 4246-4250.

[22] N. Jamali and C. Sammut, "Material classification by tactile sensing using surface textures," in Robotics and Automation (ICRA), 2010 IEEE International Conference on. IEEE, 2010, Conference Proceedings, pp. 2336-2341.

[23] _ _ "Majority voting: material classification by tactile sensing using surface texture," Robotics, IEEE Transactions on, vol. 27, no. 3, pp. $508-521,2011$

[24] J. A. Fishel and G. E. Loeb, "Bayesian exploration for intelligent identification of textures," Frontiers in Neurorobotics, vol. 6, 2012.

[25] S.-H. Kim, J. Engel, C. Liu, and D. L. Jones, "Texture classification using a polymer-based mems tactile sensor," Journal of micromechanics and Microengineering, vol. 15, no. 5, p. 912, 2005.

[26] F. De Boissieu, C. Godin, B. Guilhamat, D. David, C. Serviere, and D. Baudois, "Tactile texture recognition with a 3-axial force mems integrated artificial finger," in Robotics: Science and Systems, 2009, Conference Proceedings.

[27] A. M. Mazid and A. Ali, "Opto-tactile sensor for surface texture pattern identification using support vector machine," in Control, Automation, Robotics and Vision, 2008. ICARCV 2008. 10th International Conference on. IEEE, 2008, Conference Proceedings, pp. 1830-1835.

[28] D. S. Chathuranga, V. A. Ho, and S. Hirai, "Investigation of a biomimetic fingertip's ability to discriminate fabrics based on surface textures," in Advanced Intelligent Mechatronics (AIM), 2013 IEEE/ASME International Conference on. IEEE, 2013, Conference Proceedings, pp. 1667-1674.

[29] S. Takamuku, G. Gomez, K. Hosoda, and R. Pfeifer, "Haptic discrimination of material properties by a robotic hand," in Development and Learning, 2007. ICDL 2007. IEEE 6th International Conference on. IEEE, 2007, Conference Proceedings, pp. 1-6.

[30] J. Edwards, J. Lawry, J. Rossiter, and C. Melhuish, "Extracting textural features from tactile sensors," Bioinspiration \& Biomimetics, vol. 3, no. 3, p. 035002, 2008. 